\title{
Implementation of Tikhonov Regularization in Oil Well Liquid Level Reading Data
}

\author{
Nurdi Irianto ${ }^{1}$, Sudjati Rachmat ${ }^{1}$, Leksono Mucharam ${ }^{1} \&$ Sapto Wahyu Indratno ${ }^{2}$ \\ ${ }^{1}$ Department of Petroleum Engineering, Institut Teknologi Bandung, Indonesia \\ ${ }^{2}$ Department of Mathematics, Institut Teknologi Bandung, Indonesia \\ Correspondence: Nurdi Irianto, Laboratory of Well Stimulation, Energy Building $7^{\text {th }}$ fl., Department of \\ Petroleum Engineering, Institut Teknologi Bandung, Jl. Ganesha 10 Bandung, West Java, Indonesia, Tel: \\ 628-1840-7136. E-mail: nurdi.irianto@students.itb.ac.id
}

Received: September 5, 2017

doi:10.5539/mas.v11n10p81
Accepted: September 21, 2017 Online Published: September 27, 2017

URL: https://doi.org/10.5539/mas.v11n10p81

\begin{abstract}
In the petroleum industry, it is common practice to do survey well liquid level for monitoring well in the purpose of evaluation well production capacity, for setting performance of downhole pump. Here we proposed liquid level survey using acoustic well sounder (echosounder) equipment. The reading of liquid level in the oil well is contained noises due to some physical and mechanical condition. An idea to handle large scattered field data contains noises is smoothness method by Tikhonov regularization.
\end{abstract}

Liquid level survey is set up under shunt in well condition, to clearly monitoring liquid level rises in the well column. So, we have acquired field data reading. Beside the field data reading using echosounder tool, we also need to calculate expected liquid level, because that noises factor in the field data. The equation is generated to define calculation of liquid level increases in the well column as a function of time. The initial condition of started liquid column height $h_{0}$ and well production rate $Q_{0}$ at $t=0$ is definite. We build the Volterra integral equation of the $1^{\text {st }}$ kind for this calculation purpose.

The ill-posed problem performs in the data, needs the solution for smoothness. Tikhonov regularization (Least Squared problem) has handling this problem. Some value of regularization parameter were employed to the calculation.

This paper is an innovative idea to maximum utilization of fluid level data monitoring in the well, while the acquired data is scattered or contains error. After smoothness of the data, qualified model solution curve is fully advantage for well interpretation.

Keywords: Acoustic well echosounder, oil well liquid level, Volterra Integral Equation, ill-posed problem, discretization, Tikhonov regularization, regularization parameter

\section{Introduction}

Working on determining liquid level using mechanical wave propagation in the well by acoustic well echosounder tool was already long time applied. In an early time by Walker (1937), Jakosky (1939), Rodd (1943), and then Mc Coy (1975 \& 1985) was continuing utilized and developed this method. Rowlan, et.al., (2011) and Taylor, et,al., (2014) in the last decade employ this acoustic method for well monitoring production artificial lift.

Liquid level depth in well is read by method shooting acoustic wave to well column annulus from surface, the digital counting of mechanical wave propagation with specific velocity, and reflection at top of liquid level in well. Pressure transducer in surface of well is a strain gauge device collects reflected acoustic waves and provides signals to the computer proportional with wave's pressure.

The research scopes on monitoring wellbore liquid level on a period of time (let say 48 hours) under technical condition of the fluid flows and rises inside vertical well bore hole. Those situations, of the acquired data creates noises in a reading. In reality mostly read liquid level data versus time gives scattered curve and contains noises.

Due to the noises problem in data acquisition, this study is an innovative idea to enhance smoothness well liquid level data for petroleum engineering interpretation purpose, such as well production capacity and placing setup 
well production artificial lift. The valuable smoothness curves data makes confidence data utilization than filtering method of field data for interpretation purpose. While the echosounder data acquisition method is also economically cost effective as a technical practice, rather than lowering downhole pressure gauge in the well.

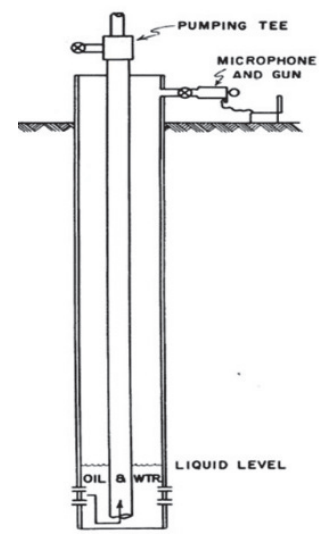

Figure 1. Measuring liquid level in well column by echometric sonic wave (modified from McCoy, et.al., 1985)

The study boundaries that the well dominantly produces liquid, and no essential amount of gas is produced from the well. Even though some wells in the field practice small gas still exist make the liquid level data is scattered.

\section{Background}

An idea to handle scattered well liquid level data contains noises is smoothness technique by Tikhonov regularization. The aim to solve problem in model estimation is governed by assessment the linear system (Golub, et.al., 1999), (Sima, et.al., 2004), (Lampe \& Voss, 2008 \& 2010), as follow,

$$
Z f=p, \quad Z \in \mathbb{R}^{m \times n}, \quad f \in \mathbb{R}^{n \times 1}, p \in \mathbb{R}^{m \times 1}, \quad m \geq n .
$$

We remember Least Squared (LS) problem,

$$
\min _{f}\|Z f-p\|_{2}
$$

Where an element of $Z$ is exact, $p$ contains noises, and $f$ is a solution.

The Tikhonov regularization and the LS problem with additional quadratic constraint, approaches ideally for solving discrete ill-posed LS problems.

For implementation the Tikhonov regularization, the equation (1) is replaced to,

$$
\min _{f}\|Z f-p\|_{2}^{2}+\lambda\|I f\|_{2}^{2},
$$

with identity matrix $I \in \mathbb{R}$ acts as a regularization matrix, or

$$
\min _{f}\|Z f-p\|_{2}^{2}+\lambda\|f\|_{2}^{2} .
$$

In the original least squares approach the system matrix $Z$ is assumed to be free from error, and all errors are confined to the observation vector $p$. And $f$ is the solution to be solved, induced by regularization parameter $\lambda$.

The matrix $Z$ comes from the discretization of an operator in an ill-posed problem and is typically very ill-conditioned.

In our study of well liquid level, the steps for regularization are ;

- First step is defining proposed model to Volterra integral equation of the $1^{\text {st }}$ kind.

- Then generate the discretization.

- Matrix operation for regularization.

- Some regularization parameter $\lambda$ is imposed. 


\section{Development of Equation, Volterra Integral Equation of the $1^{\text {st }}$ Kind}

Flow rate of liquid fills in the wellbore from reservoir can be represents as liquid level rises in well column as the following function of time,

$$
Q_{(t)}=A \frac{d h_{(t)}}{d t}
$$

Where $Q_{(t)}$ is volume rate (bbl/day) at time $t, A$ is (square inch) area of internal section vertical well pipe, $d h$ liquid rises (feet), $d t$ is time elapse (day).

From equation (4) we get a Volterra integral equation of the $1^{\text {st }}$ kind, for calculation liquid level as a function of time in following form,

$$
h_{(t)}^{c}=h_{0}+\frac{1}{A} \int_{0}^{t} Q_{(s)} d s .
$$

Where $h_{(t)}^{c}$ is calculated liquid level in feet, $d s$ is also still represent time elapse (day) in integral equation form. For initial condition, $h_{0}$ is liquid level at time $t_{0}$, we will see next $Q_{0}$ is production rate in the beginning just before start survey.

Applying the equation by parts in (5) the Volterra integral equation of the $1^{\text {st }}$ kind can be written as follow,

$$
h_{(t)}^{c}=h_{0}+\frac{1}{A} \int_{t=0}^{t} Q_{(s)} d s=h_{0}+\frac{1}{A}\left[Q_{(s)} s\right]_{t=0}^{t}-\frac{1}{A} \int_{t=0}^{t} s d Q .
$$

Let be from,

$$
f_{(t)}=\frac{d Q}{d t}
$$

From (7) we get the relation,

$$
Q_{(t)}=Q_{0}+\int_{t=0}^{t} f_{(s)} d s
$$

Then equation (6) can be written by substituting equation (7) as follow,

$$
h_{(t)}^{c}=h_{0}+\frac{1}{A} Q_{0} t-\frac{1}{A} \int_{t=0}^{t} s f_{(s)} d s .
$$

Which implies,

$$
h_{(t)}^{c}=h_{0}+\frac{1}{A} t Q_{0}+\frac{1}{A} \int_{t=0}^{t}(t-s) f_{(s)} d s
$$

One can see that (9) is a Volterra integral equation of the $1^{\text {st }}$ kind with the Kernel $K(t, s)=t-s$, which is a smooth Kernel. The smoothness of the Kernel makes problem of solving equation (9) is categorized as an ill-posed problem. There for we need a regularization method to get solution of the equation (Hansen, 1998). $K(t, s)$ is generated in the discretization process.

The discretization of the Volterra equation is given in the following section.

\section{Discretization}

Prepare for discrete expression, equation become,

$$
h_{\left(t_{i}\right)}=h_{0}+\frac{1}{A} t_{i} Q_{0}+\frac{1}{A} \sum_{j=0}^{t_{i}} \alpha_{i j}\left(t_{i}-t_{j}\right) f_{\left(t_{j}\right)} \Delta t_{j}
$$

Where $t_{j}$ is time in the data point due to discretization while interval discrete $\Delta t_{j}$ is constant, $t_{i}$ is time in field data acquisition, and $t_{i_{0}}<t_{j}<t_{i \max }$, and also $\alpha_{i j}$ are coefficients apply in the Trapezoidal or Simpson rules.

Here we apply Trapezoidal rule whit in equation (10),

$$
\sum_{j=0}^{t_{i}} \alpha_{i j}\left(t_{i}-t_{j}\right) \Delta t_{j}
$$


$\alpha_{i j}=\frac{1}{2}$ for $t_{j}$, while $j=1$ into $n-1$,

$\alpha_{i j}=1$ for $t_{0}$ and $t_{\text {max }}$

$\mathrm{i}=1,2,3, \ldots \ldots, \mathrm{n}_{\mathrm{i}}$ and $\mathrm{j}=1,2,3, \ldots, \mathrm{n}_{\mathrm{j}}$.

For next, Kernel matrix $\mathrm{Z}$ is,

$$
Z^{i \times j}=\sum_{j=0}^{t_{i}} \alpha_{i j}\left(t_{i}-t_{j}\right) \Delta t_{j}
$$

For good smoothness, try $\Delta t_{j} \ll \Delta t_{i}$.

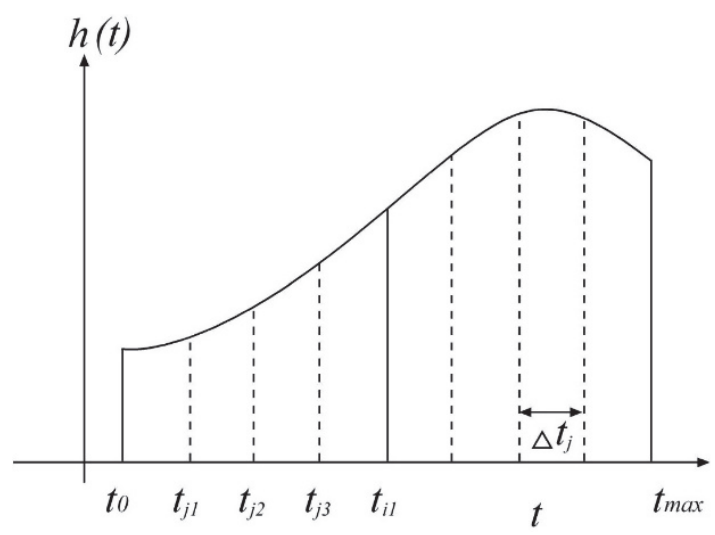

Figure 2. Interval discretization

In Figure 2., time interval of discrete is forced to smaller than time interval of data acquisition, to get better smoothness purpose.

\section{Matrix Form}

Because liquid level in the well from survey data is time dependent, we are doing matrix operation for calculation process. Then we do matrix notation for solving the algorithm.

From (10) we write the equation in the following matrix-vector forms,

$$
h_{(t)}^{c}=X h_{0}+\frac{1}{A} Y Q_{0}+\frac{1}{A} Z f
$$

Where matrix column $X=(1,1, \ldots, 1)^{T}$ and $Y=\left(t_{i_{1}}, t_{i_{2}}, \ldots . t_{i_{n}}\right)^{T}$, and squared / rectangle matrix $Z=$ $\sum_{j=0}^{t_{i}} \alpha_{i j}\left(t_{i}-t_{j}\right) \Delta t_{j}$ is tridiagonal matrix from discretization, $i=1,2, \ldots n_{\mathrm{i}}$, and $j=1,2, \ldots, n_{j}$, also $n_{j}>n_{i}$.

The unknown vector column $f$ should be found, in the regularization process.

From that matrix form equation (11), the matrix is expressed as,

$$
\left[\begin{array}{c}
h_{(t 1)}^{c} \\
h_{(t 2)}^{c} \\
\vdots \\
\vdots \\
h_{\left(t n_{i}\right)}^{c}
\end{array}\right]=\left[\begin{array}{c}
1 \\
1 \\
1 \\
\vdots \\
1
\end{array}\right] h_{0}+\frac{1}{A}\left[\begin{array}{c}
t_{1} \\
t_{2} \\
\vdots \\
\vdots \\
t_{n_{i}}
\end{array}\right] Q_{0}+\frac{1}{A}\left[\begin{array}{cccc}
\alpha_{11}\left(t_{i_{1}}-t_{j_{1}}\right) \Delta t_{j} & 0 & 0 \\
\vdots & \ddots & \ddots & 0 \\
\alpha_{n_{i} 1}\left(t_{n_{i}}-t_{j_{1}}\right) \Delta t_{j} & \alpha_{n_{i} 2}\left(t_{n_{i}}-t_{j_{2}}\right) \Delta t_{j} & \ddots & \cdots \\
\ldots
\end{array}\right] f
$$

Determine $f$ to calculate expected $\hat{h}$ or $h_{i}^{c}$.

If we can calculate $p$ as,

$$
p=\left[\begin{array}{cccc}
\alpha_{11}\left(t_{i_{1}}-t_{j_{1}}\right) \Delta t_{j} & 0 & 0 & 0 \\
\vdots & \ddots & \ddots & 0 \\
\alpha_{n_{i} 1}\left(t_{n_{i}}-t_{j_{1}}\right) \Delta t_{j} & \alpha_{n_{i} 2}\left(t_{n_{i}}-t_{j_{2}}\right) \Delta t_{j} & \ldots & 0
\end{array}\right] f=Z f
$$


Where is,

$$
p=A\left(\left[\begin{array}{c}
h_{(t 1)}^{c} \\
h_{(t 2)}^{c} \\
\vdots \\
\vdots \\
h_{\left(t_{n_{i}}\right)}^{c}
\end{array}\right]-\left[\begin{array}{c}
1 \\
1 \\
1 \\
\vdots \\
1
\end{array}\right] h_{0}-\frac{1}{A}\left[\begin{array}{c}
t_{1} \\
t_{2} \\
\vdots \\
\vdots \\
t_{n_{i}}
\end{array}\right] Q_{0}\right)
$$

$p=Z f$ the columns of $Z$ is very large then rows of $f$, so matrix multiplication cannot work. To finalize that work, we will describe below.

\section{Implementation of Tikhonov Regularization}

Recall, the linear systems,

$$
Z f=p \quad Z \in \mathbb{R}^{m \times n} p \in \mathbb{R}^{m x 1} \quad m \geq n .
$$

In the classical least squares approach the system matrix $Z$ is assumed to be free from error, and all errors are confined to the observation vector $p$. And $f$ is the solution to be solved by regularization.

Let our propose equation model is not simple linear as (11). Then we make it to be simple linear as,

$$
Z f=p \text {. }
$$

Because $p$ contains exact field data survey $h_{\left(t_{i}\right)}^{m}$, that $p$ also constant, then allocate vector $f$ as a left hand side to be proposed as a solution.

Because linear system for regularization required condition, $Z \in \mathbb{R}^{m \times n}$. In our study, condition of Kernel vector $Z$ from the integral equation in fact that $n_{i}<n_{j}$, so we have to do operation similar condition as $m>n$, by multiplying Kernel $Z$ with his transpose $Z^{T}$ become,

$$
f=\left(Z^{T} Z\right)^{-1} Z^{T} p
$$

Since the original equation has ill-posed problem, the discrete version also give ill condition linear system (Indratno \& Ramm, 2009), therefor (12) will be unstable. We propose the Thikonov regularization as follows,

$$
\min _{f}\left[\|Z f-p\|_{2}^{2}+\lambda\|f\|_{2}^{2}\right]
$$

where $\lambda>0, \lambda \in \mathbb{R}$ is regularization parameter.

The solution (13) is of the following form,

$$
f_{\lambda}=\left(Z^{T} Z+\lambda I\right)^{-1} Z^{T} p
$$

After we determined vector column $f_{\lambda}$, put the solution into matrix operation represents our integral equation, to calculated expected model liquid level.

$$
h_{\lambda}^{c}=X h_{0}+\frac{1}{A} Y Q_{0}+\frac{1}{A} Z f_{\lambda}
$$

\section{Data Processing}

Field data acquisition of liquid level reading by Echometer equipment is prepared for discretization. For example, liquid level well 92S-5, there are 100 times field data reading for about 48 jam survey in well. In purpose to discretization, we make constant partition $\Delta t_{j}$ on the field data $t_{i}$, let the data we discretize into 1000 points $\left(\Delta t_{j}=t_{i \text { max }}-t_{i_{0}}\right) / 999$. Create Kernel matrix $Z^{i x j}$ from discretization process, where $n_{i}<<n_{j}$ to smoothness as possible, and of course $\Delta t_{j}<\Delta t_{i}$. So, the matrix become $Z^{100 \times 1000}$. We remember, in the theory of linear system (1), the row component of Kernel matrix should be the same or larger than the column in the matrix $(m>n)$. For this purpose we do multiplication matrix $Z$ from discretization result with his transpose $Z^{T}$, before we impose the regularization parameter.

We do matrix calculation to define unknown vector column $f$ as a solution, the method was described above. The calculation of $f$ contains ill condition. To satisfy the calculation, apply regularization parameter $\lambda$, then we get the solution $f_{\lambda}$. 


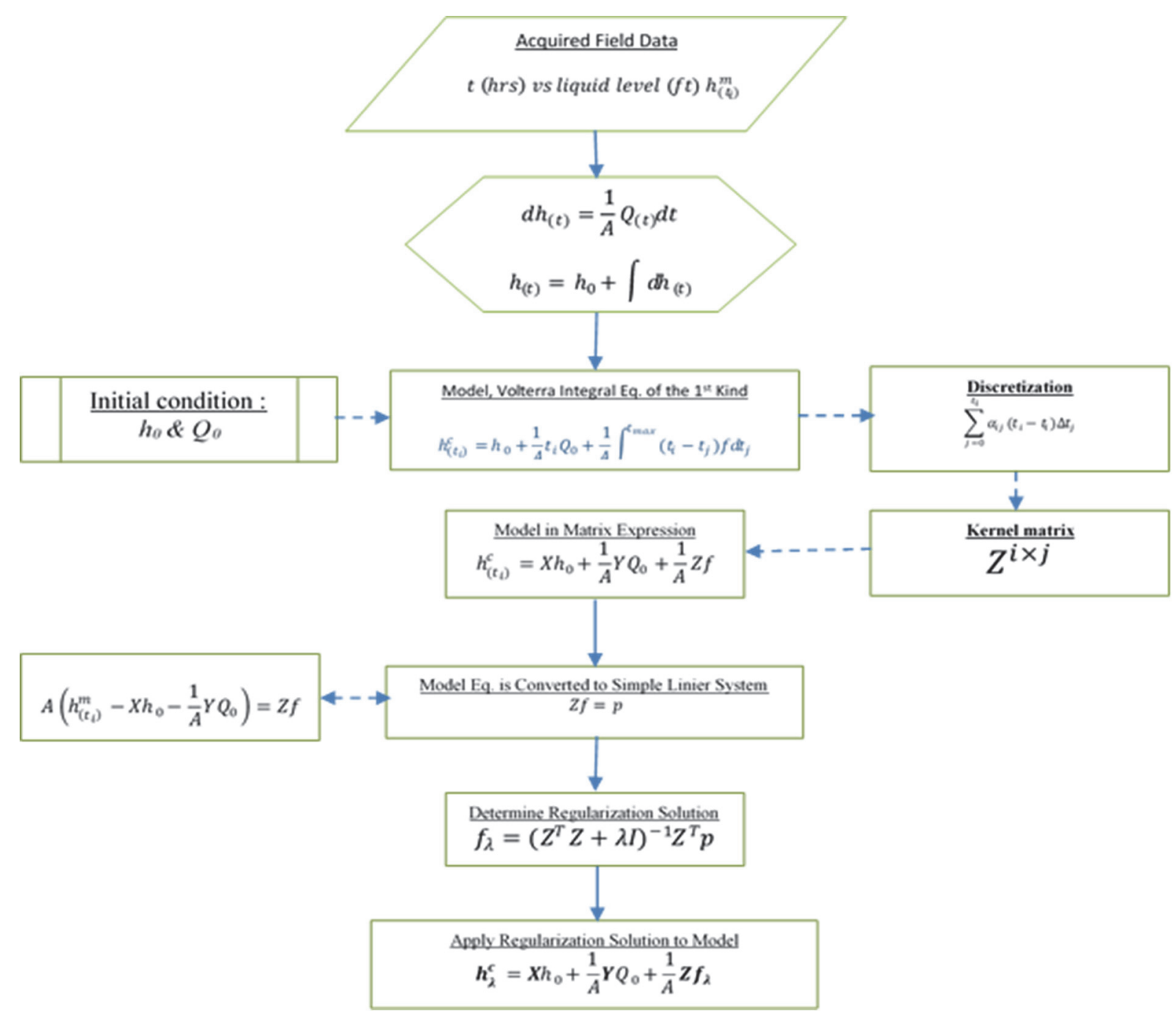

Figure 3. Flowchart to get regularized model calculated of well liquid level

The solution $f_{\lambda}$ apply to the propose model liquid level calculation (15). Use other some values of parameter $\lambda$ to get the best model of calculated liquid level.

The process of calculation to get regularization solution for model calculated of liquid level in well is summarized in the flow chart.

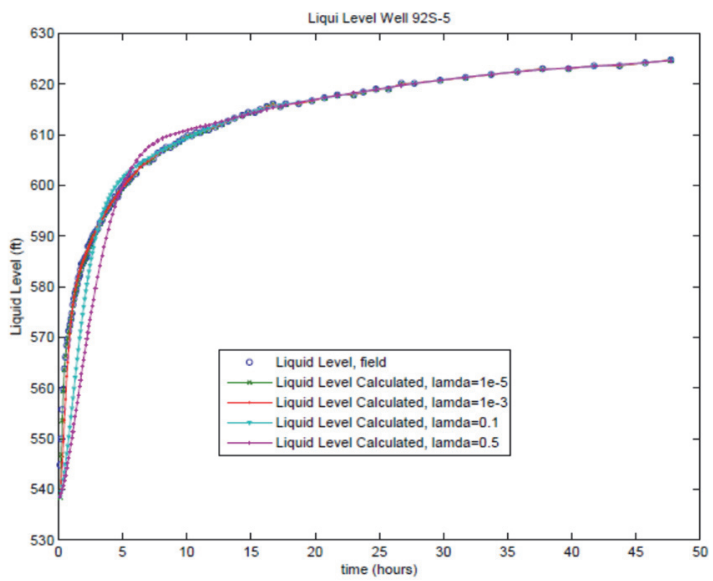

Figure 4. Well 92S-5 liquid level rises vs time, with some number of regularization parameter. 


\section{Result and Discussion}

This paper reports model calculation for some oil wells of the field in Indonesia. The well is simple vertical, dominantly produce liquid.

Implementation some number of regularization parameter in well liquid level data indicates two schemes. First while the field data acquired is mostly scattered larger number of regularization parameter $(\lambda=0.5,0.8,5,8$ etc. $)$ give smoothness curve to the middle of data, see the graphics of calculated model for well 2D-8 (Figure 5.), 18D-6 (Figure 7.), and well 81D-7 (Figure 9.). For well with acquired data less scattered or almost ideal smooth, the large regularization parameter make the model solution deviated from the field data. See well 92S-5 (Figure 4.) smaller $\lambda<0.1$ show the calculated solution match to the field data, but when the parameter bigger $\lambda=0.1$, and 0.5 the model solution make the curve deviated from the original field curve (points).

While we approach the smallest number of regularization parameter $\lambda=1 \times 10^{-5}$, and $1 \times 10^{-17}$ see well 2D-8 (Figure 5. \& Figure 6.), $\lambda=1 \times 10^{-7}$ in well 81D-7 (Figure 9.), it forces the calculated model closes to the field data. We understand when $\lambda \approx 0$ regularization parameter almost close to zero, the calculation become similar to ill-posed condition, means looks no regularization parameter approached for the solution.

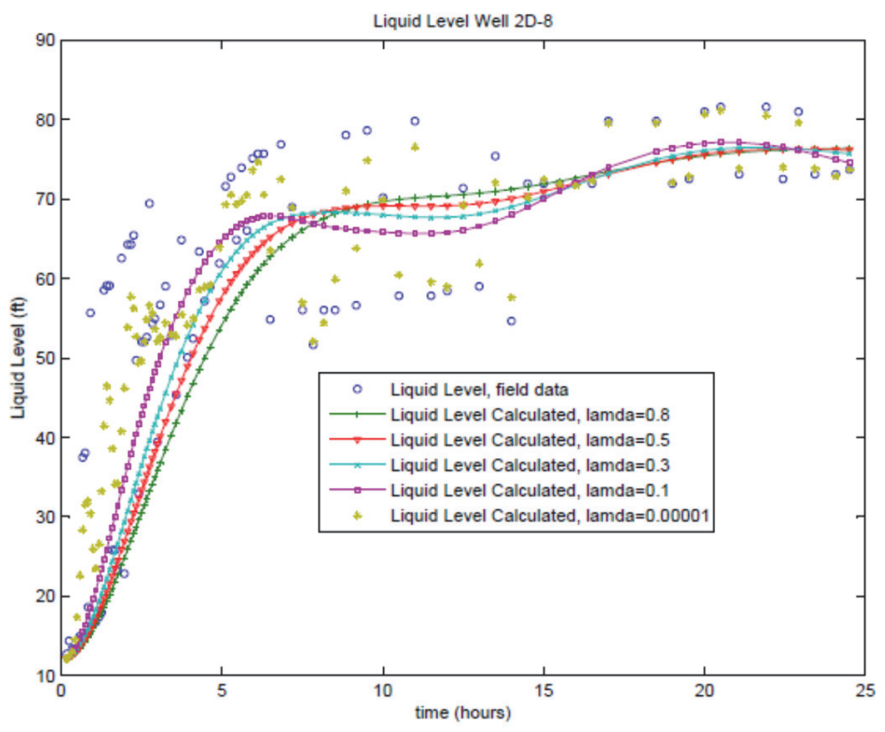

Figure 5. Well 2D-8 liquid level rises vs time, with some number of regularization parameter

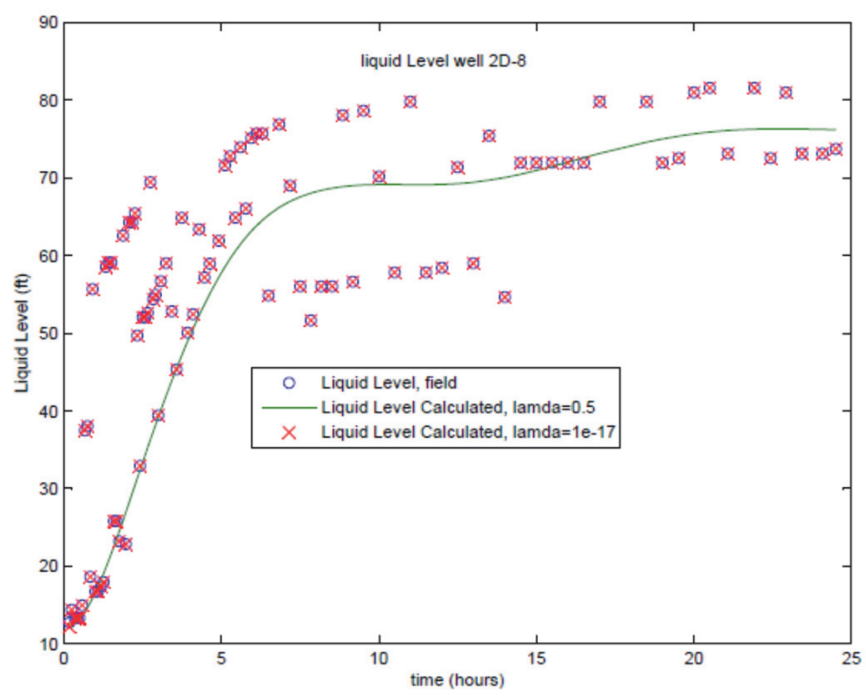

Figure 6. Well 2D-8 liquid level rises vs time, with very small value of regularization parameter $\lambda=1 \times 10^{-17}$ (red cross) 


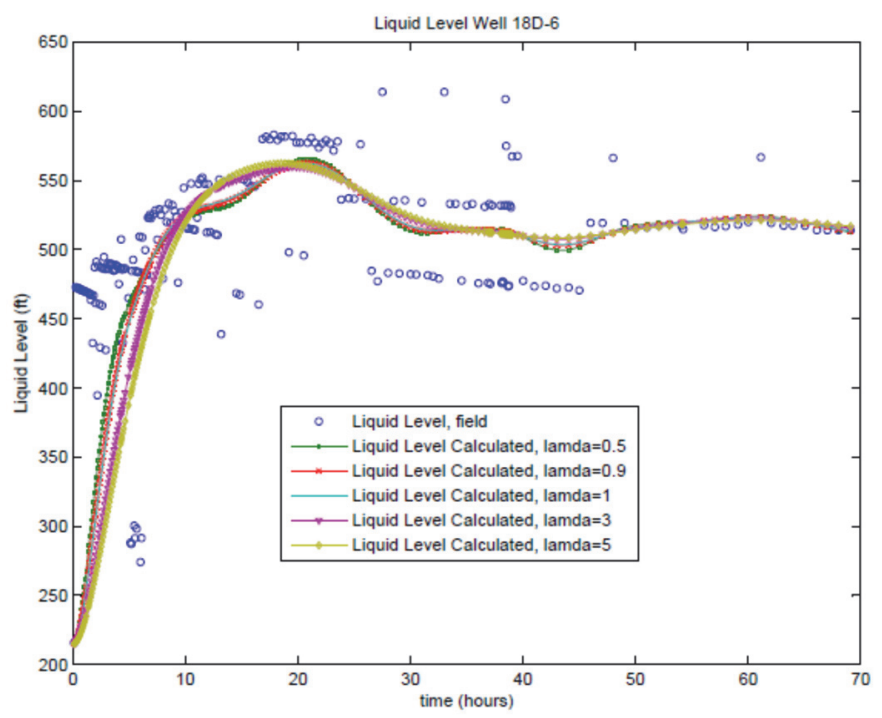

Figure 7. Well 18D-6 liquid level rises vs time, with some number of regularization parameter

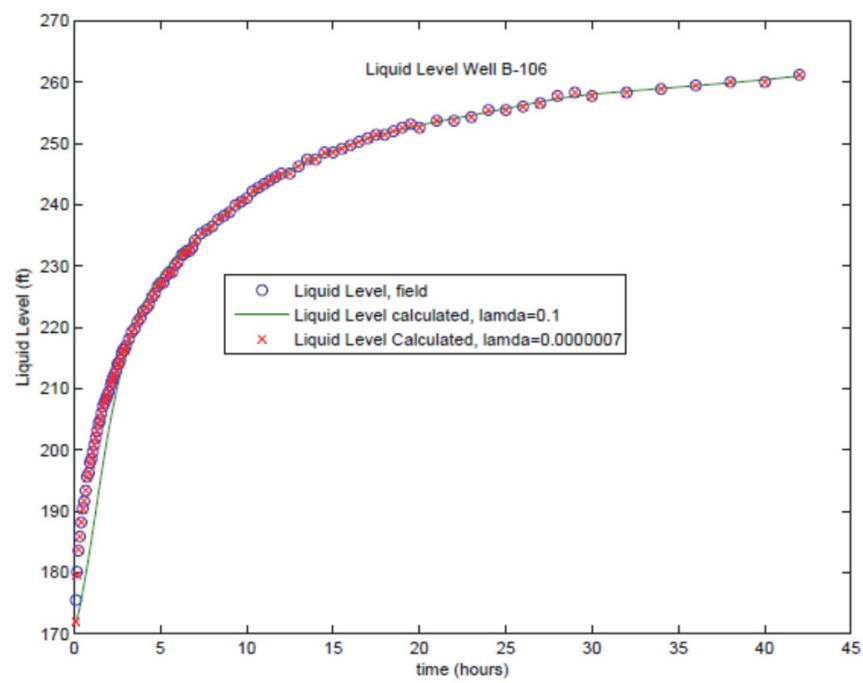

Figure 8. Well B-106 liquid level rises vs time, with some number of regularization parameter

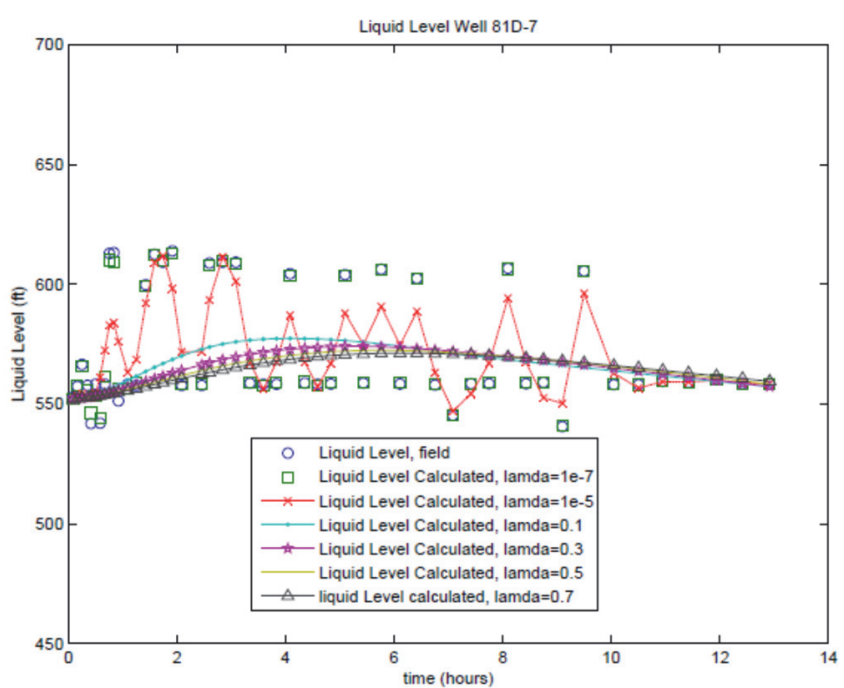

Figure 9. Well 81D-7 liquid level rises vs time, with some number of regularization parameter 
Table 1. Smoothness of calculated model solution related to regularization parameter $\lambda$

\begin{tabular}{ccc}
\hline Well & Lambda $(\lambda)$ & Calculated Curve / Points \\
\hline 92 S-5 & $1 \times 10^{-5}$ & Scattered \\
& $1 \times 10^{-3}$ & Smooth \\
& 0.1 & Smooth deviated \\
2D-8 & 0.5 & Smooth deviated \\
& $1 \times 10^{-17}$ & Scattered and match to fields data points \\
& $1 \times 10^{-5}$ & Scattered \\
& 0.1 & Smooth wiggled \\
& 0.3 & Smooth wiggled \\
& 0.5 & Smooth \\
18 D-6 & 0.8 & Smooth deviated \\
& 0.5 & Smooth wiggled \\
& 0.9 & Smooth wiggled \\
& 1 & Smooth less wiggled \\
& 3 & Smooth \\
B-106 & $7 \times 10^{-7}$ & Smooth almost deviated \\
& 0.1 & Match to field data \\
$81 D-7$ & $1 \times 10^{-7}$ & Smooth \\
& $1 \times 10^{-5}$ & Almost match to field data \\
& 0.1 & Large scattered \\
& 0.3 & Smooth almost to mid data \\
& 0.5 & Smooth to lower data \\
& 0.7 & Smooth to lower data \\
\end{tabular}

\section{Conclusion}

We have two group of well liquid level field data, first is the scattered data while the data hard to be utilized for interpretation without smoothness, and the second the field data almost not scattered.

The regularization process on the scattered field data, to direct calculated model solution, summaries as ;

Small regularization parameter, make the calculation result closes to the field data acquired, $\lambda=1 \times 10^{-5}$, $\lambda=1 \times 10^{-7}$, we can see very clear when we put $\lambda=1 \times 10^{-17}$ on well $2 \mathrm{D}-8$ the model calculated is almost matched with field data. On this very small parameter of $\lambda$ the regularization is classified as over determined.

Large regularization parameter, make the calculation generalized the field data acquired, $\lambda=0.8, \lambda>1, \lambda=5$. The regularization is called under-estimated or less projected. But large $\lambda$ give more smoothness figures from the scattered field data, make the data is easier utilization for interpretation purpose.

To get smoothness, large data in the discrete or small discrete interval $\left(\Delta t_{j}\right)$, very support to the smoothness.

\section{References}

Golub, G. H., Hansen, P. C., \& O’leary, D. P. (1999). Tikhonov Regularization and Total Least Squares. Society for Industrial and Applied Mathematics (SIAM). Journal on Matrix Analysis and Application, 21(1), 185194. https://doi.org/10.1137/S0895479897326432

Hansen, P. C. (1998). Rank-deficient and Discrete Ill-posed Problems. Technical University of Denmark, Lyngby, Denmark, eISBN: 978-0-89871-969-7, Society for Industrial and Applied Mathematics (SIAM). https://doi.org/10.1137/1.9780898719697

Indratno, S. W., \& Ramm, A. G. (2009). An Iterative Method for Solving Fredholm Integral Equations of the First Kind. Department of Mathematics Kansas State University. Manhattan. USA. Article in International Journal of Computing Science and Mathematics. November 2009, arXiv:0911.3071v2 [math.NA] 4 Dec 2009, https://doi.org/10.1504/IJCSM.2009.030913

Indratno, S. W., \& Ramm, A. G. (2009). Dynamical Systems Method for Solving Ill-conditioned Linear Algebraic Systems. Department of Mathematics Kansas State University. Manhattan. USA. Article in International Journal of Computing Science and Mathematics. November 2009. arXiv:0911.3065v2 [math.NA] 4 Dec 2009. https://doi.org/10.1504/IJCSM.2009.030911 
Jakosky, J. J. (1939). Bottom-hole Measurements in Pumping Wells. International Geophysics Inc. (Trans. AIME) SPE Journal Paper. SPE-939062-G. https://doi.org/10.2118/939062-G_

Krawczyk-Stando, D., \& Rudnicki, M. (2007). Regularization Parameter Selection in Discrete Ill-posed Problem - The Use of The U-curve. International Journal Applied Mathematic Computer Science, 17(2), 157-164. Poland. https://doi.org/10.2478/v10006-007-0014-3

Lampe, J. (2010). Solving Regularized Total Least Squares Problems Based on Eigen Problems. (Dissertation. Vom Promotionsausschuss der Technischen Universität Hamburg - Harburg zur Erlangung des Akademischen Grades Doktor der Naturwissenschaften). Retrieved from http://www.dissertation.de

Lampe, J., \& Voss, H. (2008). A Fast Algorithm for Solving Regularized Total Least Squares Problems. Institute of Numerical Simulation. Hamburg University of Technology. D-21071 Hamburg. Germany. Electronic Transactions on Numerical Analysis, 31, 12-24. Kent State University. ISSN 1068-9613.

Lampe, J., \& Voss, H. (2010). Solving Regularized Total Least Squares Problems Based on Eigen Problems. Institute of Numerical Simulation. Hamburg University of Technology. D-21071 Hamburg. Germany. Taiwanese Journal of Mathematics. Vol. 14. No. 3A. p. 885-909. Retrieved from http://www.tjm.nsysu.edu.tw/

McCoy, \& James, N. (1975). Analyzing Well Performance VII. SPE Oklahoma City Regional Meeting 24-25 March. Oklahoma City. Oklahoma. SPE-5403-MS Society of Petroleum Engineers Conference Paper. https://doi.org/10.2118/5403-MS

McCoy, J. N., Podio, A. L., Huddleston, K. L., \& Drake, B. (1985). Acoustic Static Bottomhole Pressures. SPE-13810-MS. SPE Production Operations Symposium. 10-12 March. Oklahoma. USA. Society of Petroleum Engineers. https://doi.org/10.2118/13810-MS

Podio, A. L., \& Mc-Coy, J. N. (1990). Computerized Well Analysis. SPE-21174-MS. SPE Latin American Petroleum Engineering Conference. Rio de Janeiro. October 14-19. Society of Petroleum Engineers. https://doi.org/10.2118/21174-MS

Rodd, C. C. (1943). Determination of Oil-well Capacities from Liquid-level Data. Gulf Oil Corp., SPE-943048-G. (Trans. AIME) SPE Journal Paper. https://doi.org/10.2118/943048-G

Rowlan, O. L., \& McCoy, J. N., (Echometer Company), Podio, A. L., (University of Texas at Austin). (2011). Pump Intake Pressure Determined from Fluid Levels, Dynamometers, and Valve-Test Measurements. SPE-142862-PA. Journal of Canadian Petroleum Technology. https://doi.org/10.2118/142862-PA

Sima, D. B., Huffel, S. V., \& Golub, G. H. (2004). Regularized Total Least Squares Based on Quadratic Eigenvalue Problem Solvers. Departement Elektrotechniek Katholieke Universiteit Leuven. Belgium. BIT Numerical Mathematics. 44, 793 - 812. Kluwer Academic Publishers Springer.

Taylor, C., Rowlan, L., \& McCoy, J. (2014). Acoustic Techniques to Monitor and Troubleshoot Gas-Lift Wells. Echometer Co. SPE-169536-MS. SPE Western North American and Rocky Mountain Joint Meeting 17-18 April. Denver. Colorado. Society of Petroleum Engineers. https://doi.org/10.2118/169536-MS

Walker, C. P. (1937). Determination of Fluid Level in Oil Wells bv the Pressure Wave Echo Method. The Depthograph Co. (Trans. AIME) SPE-937032-G. SPE Journal Paper. https://doi.org/10.2118/937032-G

\section{Copyrights}

Copyright for this article is retained by the author(s), with first publication rights granted to the journal.

This is an open-access article distributed under the terms and conditions of the Creative Commons Attribution license (http://creativecommons.org/licenses/by/4.0/). 